Supporting Information

Synthesis of novel 1,2,3-triazole-dihydro[3,2-c]chromenones as acetylch acetylcholinesterase inhibitors

Mina Saeedi, Shirin Ansari, Mohammad Mahdavi, Reyhaneh Sabourian, Tahmineh Akbarzadeh, Alireza Foroumadi, and Abbas Shafiee 
Synthesis of 4-(prop-2-yn-1-yloxy)benzaldehyde 3: A solution of 4-hydroxybenzaldehyde $1(1 \mathrm{mmol})$ and propargyl bromide $2(1 \mathrm{mmol})$ in DMF $(8 \mathrm{~mL})$ was heated at $80^{\circ} \mathrm{C}$ for $12 \mathrm{~h}$. After completion of reaction, the precipitated product was filtered off, washed with water, dried at $50{ }^{\circ} \mathrm{C}$, and used for further reaction.

\section{Synthesis of 2-amino-5-oxo-4-(4-(prop-2-yn-1-yloxy)phenyl)-4,5-dihydropyrano[3,2-}

c]chromene-3-carbonitrile 6: A solution of 4-(prop-2-yn-1-yloxy)benzaldehyde 3 (1 mmol), 4-hydroxycoumarine 4 (1 mmol), malonitrile 5 (1 mmol), and DBU (1 mmol) was heated at room temperature for $7 \mathrm{~h}$. After completion of reaction, the precipitated product was filtered off, washed with cold ethanol, dried at $50{ }^{\circ} \mathrm{C}$, and used for further reaction.

Synthesis of azide derivatives 9: A mixture of benzyl bromide or chloride derivative 7 (1 mmol) and sodium azide $8(1 \mathrm{mmol})$ was stirred at $80{ }^{\circ} \mathrm{C}$ for $3-5 \mathrm{~h}$. After completion of reaction, the precipitated product was filtered off, washed with water, dried at $50{ }^{\circ} \mathrm{C}$, and used for the next reaction.

\section{Synthesis of 1,2,3-triazole-dihydro[3,2-c]chromenone derivatives 10: General}

Procedure: A solution of 2-amino-5-oxo-4-(4-(prop-2-yn-1-yloxy)phenyl)-4,5dihydropyrano[3,2-c]chromene-3-carbonitrile 6 (1 mmol), azide derivative 9 (1 mmol), and $\mathrm{CuI}(5 \mathrm{~mol} \%)$ in DMF $(8 \mathrm{~mL})$ was stirred at $80{ }^{\circ} \mathrm{C}$ for $4-5 \mathrm{~h}$. After completion of reaction, the mixture was cooled down to room temperature. Then, water $(25 \mathrm{~mL})$ was added to the mixture and the organic phase was extracted using ethyl acetate $(3 \times 25)$. It was dried over $\mathrm{Na}_{2} \mathrm{SO}_{4}$ and the solvent was evaporated under vacuum. Crud product was recrystallized from ethanol to give pure product $\mathbf{1 0}$.

2-Amino-4-(4-((1-(4-methylbenzyl)-1H-1,2,3-triazol-4-yl)methoxy)phenyl)-5-oxo-4H,5Hpyrano[3,2-c]chromene-3-carbonitrile (10a). 
Yield: $80 \%$, white crystals, mp $230-232{ }^{\circ} \mathrm{C}$. IR (KBr): 3351, 3320, 3079, 29551, 2197, 1715 , 1673, 1606, $1507 \mathrm{~cm}^{-1} .{ }^{1} \mathrm{H}$ NMR (500 MHz: DMSO-d 6 ): 2.26 (s, 3H, $\left.\mathrm{CH}_{3}\right), 4.40$ (s, 1H, H4), $5.11\left(\mathrm{~s}, 2 \mathrm{H}, \mathrm{CH}_{2}\right), 5.71\left(\mathrm{~s}, 2 \mathrm{H}, \mathrm{CH}_{2}\right), 6.95\left(\mathrm{~d}, J=8.8 \mathrm{~Hz}, 2 \mathrm{H}, \mathrm{H} 3{ }^{\prime}, \mathrm{H} 5^{\prime}\right), 7.11-7.22(\mathrm{~m}, 6 \mathrm{H}$, H2', H6', H2", H3", H5", H6"), 7.39 (s, 2H, NH $), 7.45$ (d, J = 8.0 Hz, 1H, H7), 7.48 (t, J= $8.0 \mathrm{~Hz}, 1 \mathrm{H}, \mathrm{H} 9), 7.70$ (td, $J=8.0,1.5 \mathrm{~Hz}, 1 \mathrm{H}, \mathrm{H} 8), 7.89$ (d, $J=8.0 \mathrm{~Hz}, 1 \mathrm{H}, \mathrm{H} 10), 8.25$ (s, 1H, triazole). ${ }^{13} \mathrm{C}$ NMR (125 MHz: DMSO-d ${ }_{6}$ ): 20.7 (Me), 36.2 (C4), 52.7 (benzyl $\mathrm{CH}_{2}$ ), $58.2(\mathrm{C} 3), 61.1\left(\mathrm{OCH}_{2}\right), 104.3$ (4H-pyran), 113.0 (C3' and 5'), 114.6 (coumarin), 116.6 (coumarin), $119.4(\mathrm{CN}), 122.5$ (triazole), 124.6 (Ar), 124.7 (Ar), 128.1 (Ar), 128.8 (Ar), 129.4 (Ar), 132.9 (Ar), 133.0 (Ar), 135.8 (Ar), 137.6 (Ar), 143.0 (triazole), 152.1 (C4'), 153.2 (coumarin, C-O), 157.2 (C2), 158.0 (4H-pyran, C-O), 159.6 (C=O (C5)). Anal. calcd. for $\mathrm{C}_{30} \mathrm{H}_{23} \mathrm{~N}_{5} \mathrm{O}_{4}$ : C, 69.62; H, 4.48; N, 13.53. Found: C, 69.80; H, 4.32; N, 13.71 .

\section{2-Amino-4-(4-((1-(2-methylbenzyl)-1H-1,2,3-triazol-4-yl)methoxy)phenyl)-5-oxo-4H,5H-} pyrano[3,2-c]chromene-3-carbonitrile (10b)

Yield: $75 \%$, white crystals, mp 207-209 ${ }^{\circ} \mathrm{C}$. IR (KBr): 3351, 3320, 2909, 2197, 1714, 1675, $1606 \mathrm{~cm}^{-1} .{ }^{1} \mathrm{H}$ NMR (500 MHz: DMSO-d $\left.)_{6}\right): 2.29$ (s, 3H, $\mathrm{CH}_{3}$ ), 4.40 9s, 1H, H4), 5.10 (s, 2H, $\left.\mathrm{CH}_{2}\right), 5.54\left(\mathrm{~s}, 2 \mathrm{H}, \mathrm{CH}_{2}\right), 5.88(\mathrm{~s}, 1 \mathrm{H}, \mathrm{H} 4), 6.60(\mathrm{~d}, J=8.0 \mathrm{~Hz}, 1 \mathrm{H}, \mathrm{H} 4 "), 6.66(\mathrm{t}, J=8.0 \mathrm{~Hz}$, 1H, H5"), 7.20 (t, J = 7.7 Hz, 1H, H9), 7.19-7.37 (m, 9H, H2', H3', H5', H6', H6", H7, H8, $\mathrm{NH}_{2}$ ), 7.66 (dd, $\left.J=7.7 \mathrm{~Hz}, 1 \mathrm{H}, \mathrm{H} 10\right), 8.05$ (s, $1 \mathrm{H}$, triazole). Anal. calcd. for $\mathrm{C}_{30} \mathrm{H}_{23} \mathrm{~N}_{5} \mathrm{O}_{4}: \mathrm{C}$, 69.62; H, 4.48; N, 13.53. Found: C, 69.51; H, 4.50; N, 13.68.

\section{2-Amino-4-(4-((1-(2-chlorobenzyl)-1H-1,2,3-triazol-4-yl)methoxy)phenyl)-5-oxo-4,5-} dihydropyrano[3,2-c]chromene-3-carbonitrile (10c)

Yield: $82 \%$, white crystals, mp 142-144 ${ }^{\circ} \mathrm{C}$. IR (KBr): 3318, 3184, 2195, 1719, 1673, 1605 $\mathrm{cm}^{-1} .{ }^{1} \mathrm{H}$ NMR (500 MHz: DMSO-d $): 4.40(\mathrm{~s}, 1 \mathrm{H}, \mathrm{H} 4), 5.11\left(\mathrm{~s}, 2 \mathrm{H}, \mathrm{CH}_{2}\right), 5.70\left(\mathrm{~s}, 2 \mathrm{H}, \mathrm{CH}_{2}\right)$, 6.96 (d, J = 8.4 Hz, 2H, H3', H5'), 7.17-7.21 (m, 3H, H2', H6', H6"), 7.33-7.38 (m, 4H, H4", 
H5", $\mathrm{NH}_{2}$ ), 7.44-7.50 (m, 3H, H7, H9, H3"), 7.70 (t, J= $\left.7.8 \mathrm{~Hz}, 1 \mathrm{H}, \mathrm{H} 8\right), 7.89$ (d, J= $7.8 \mathrm{~Hz}$, 1H, H10), 8.24 (s, $1 \mathrm{H}$, triazole). ${ }^{13} \mathrm{C}$ NMR (125 MHz: DMSO-d $\left.d_{6}\right): 36.1$ (C4), 50.5 (benzyl $\left.\mathrm{CH}_{2}\right), 58.0(\mathrm{C} 3), 60.9\left(\mathrm{OCH}_{2}\right), 104.1$ (4H-pyran), 113.0 (C3' and 5'), 114.5 (coumarin), 116.5 (coumarin), $119.2(\mathrm{CN}), 122.4$ (triazole), 124.6 (Ar), 125.0 (Ar), 127.6 (Ar), 128.7 (Ar), 129.5 (Ar), 130.1 (Ar), 130.4 (Ar), 132.5 (Ar), 132.8 (Ar), 133.1 (Ar), 135.7 (Ar), 142.8 (triazole), 152.0 (C4'), 153.0 (coumarin, C-O), 157.0 (C2), 157.8 (4H-pyran, C-O), 159.4 $(\mathrm{C}=\mathrm{O}(\mathrm{C} 5))$. Anal. calcd. for $\mathrm{C}_{29} \mathrm{H}_{20} \mathrm{ClN}_{5} \mathrm{O}_{4}: \mathrm{C}, 64.75 ; \mathrm{H}, 3.75 ; \mathrm{N}, 13.02$. Found: $\mathrm{C}, 64.87 ; \mathrm{H}$, $3.59 ; \mathrm{N}, 12.87$.

\section{2-Amino-4-(4-((1-(2,3-dichlorobenzyl)-1H-1,2,3-triazol-4-yl)methoxy)phenyl)-5-oxo- 4H,5H-pyrano[3,2-c]chromene-3-carbonitrile (10d)}

Yield: $77 \%$, white crystals, mp $136-137{ }^{\circ} \mathrm{C}$. IR (KBr): 3321, 3184, 2909, 2195, 1719, 1672, $1605 \mathrm{~cm}^{-1} .{ }^{1} \mathrm{H}$ NMR (500 MHz: DMSO-d $)$ ) 4.36 (s, 1H, H4), 5.08 (s, 2H, $\left.\mathrm{CH}_{2}\right), 5.72$ (s, 2H, $\mathrm{CH}_{2}$ ), 6.95 (d, $J=8.5 \mathrm{~Hz}, 2 \mathrm{H}, \mathrm{H} 3$ ', H5'), 7.09 (d, $\left.J=7.2 \mathrm{~Hz}, 1 \mathrm{H}, \mathrm{H} 6 "\right) 7.14$ (d, $J=8.5 \mathrm{~Hz}$, 2H, H2', H6'), 7.32-7.48 (m, 3H, H5", $\mathrm{NH}_{2}$ ), 7.42 (d, $\left.J=7.5 \mathrm{~Hz}, 1 \mathrm{H}, \mathrm{H} 7\right), 7.46$ (t, $J=7.5 \mathrm{~Hz}$, 1H, H9), 7.61 (d, J=7.2 Hz, 1H, H4"), 7.67 (t, $J=7.5 \mathrm{~Hz}, 1 \mathrm{H}, \mathrm{H} 8), 7.85$ (d, $J=7.5 \mathrm{~Hz}, 1 \mathrm{H}$,

H10), 8.24 (s, 1H, triazole). ${ }^{13} \mathrm{C}$ NMR (125 MHz: DMSO-d $)$ ): 36.1 (C4), 48.6 (benzyl $\mathrm{CH}_{2}$ ), $58.1(\mathrm{C} 3), 60.9\left(\mathrm{OCH}_{2}\right), 104.2$ (4H-pyran), 113.0 (C3' and 5'), 114.6 (coumarin), 116.5 (coumarin), $119.2(\mathrm{CN}), 122.4$ (triazole), 124.6 (Ar), 124.9 (Ar), 128.7 (Ar), 128.9 (Ar), 129.0 (Ar), 130.3 (Ar), 131.4 (Ar), 131.6 (Ar), 132.8 (Ar), 135.7 (Ar), 135.9 (Ar), 142.5 (triazole), 152.1 (C4'), 153.1 (coumarin, C-O), 157.1 (C2), 157.9 (4H-pyran, C-O), 159.5 $(\mathrm{C}=\mathrm{O}(\mathrm{C} 5))$. Anal. calcd. for $\mathrm{C}_{29} \mathrm{H}_{19} \mathrm{Cl}_{2} \mathrm{~N}_{5} \mathrm{O}_{4}: \mathrm{C}, 60.85 ; \mathrm{H}, 3.35 ; \mathrm{N}, 12.24$. Found: $\mathrm{C}, 60.63$; H, 3.47; N, 12.36 .

\section{2-Amino-4-(4-((1-(2,4-dichlorobenzyl)-1H-1,2,3-triazol-4-yl)methoxy)phenyl)-5-oxo-} 4H,5H-pyrano[3,2-c]chromene-3-carbonitrile (10e) 
Yield: $70 \%$, white crystals, mp $231-233{ }^{\circ} \mathrm{C}$. IR (KBr): 3400, 3308, 3132, 2206, 1714, 1675 , $1608 \mathrm{~cm}^{-1} .{ }^{1} \mathrm{H}$ NMR (500 MHz: DMSO-d $)$ ) 4.40 (s, 1H, H4), 5.11 (s, 2H, $\left.\mathrm{CH}_{2}\right), 5.71(\mathrm{~s}, 2 \mathrm{H}$, $\mathrm{CH}_{2}$ ), 6.96 (d, J = 8.7 Hz, 2H, H3', H5'), 7.17-7.21 (m, 3H, H2', H6', H6"), 7.33-7.51 (m, 6H, H7, H9, H3", H5", NH $), 7.71$ (td, $J=7.9,1.5 \mathrm{~Hz}, 1 \mathrm{H}, \mathrm{H} 8), 7.89$ (dd, $J=7.9,1.5 \mathrm{~Hz}, 1 \mathrm{H}$, H10), 8.25 (s, 1H, triazole). ${ }^{13} \mathrm{C}$ NMR (125 MHz: DMSO-d $\left.)_{6}\right): 36.1$ (C4), 50.6 (benzyl $\mathrm{CH}_{2}$ ), $58.1(\mathrm{C} 3), 61.0\left(\mathrm{OCH}_{2}\right), 104.2$ (4H-pyran), $113.0\left(\mathrm{C}^{\prime}\right.$ and 5'), 114.6 (coumarin), 116.6 (coumarin), $119.3(\mathrm{CN}), 122.4$ (triazole), 122.5 (Ar), 124.7 (Ar), 125.1 (Ar), 127.7 (Ar), 128.8 (Ar), 129.6 (Ar), 130.4 (Ar), 132.6 (Ar), 132.9 (Ar), 133.2 (Ar), 135.8 (Ar), 142.9 (triazole), 152.1 (C4'), 153.1 (coumarin, C-O), 157.1 (C2), 157.9 (4H-pyran, C-O), 159.5 $(\mathrm{C}=\mathrm{O}(\mathrm{C} 5))$. Anal. calcd. for $\mathrm{C}_{29} \mathrm{H}_{19} \mathrm{Cl}_{2} \mathrm{~N}_{5} \mathrm{O}_{4}$ : C, 60.85; H, 3.35; N, 12.24. Found: $\mathrm{C}, 60.73$; H, 3.28; N, 12.11 .

\section{2-Amino-4-(4-((1-(2,6-dichlorobenzyl)-1H-1,2,3-triazol-4-yl)methoxy)phenyl)-5-oxo- 4H,5H-pyrano[3,2-c]chromene-3-carbonitrile (10f)}

Yield: 70\%, white crystals, mp 162-164 ${ }^{\circ} \mathrm{C} . \mathrm{IR}(\mathrm{KBr}): 3430,3329,3180,2196,1723,1673$, $1605 \mathrm{~cm}^{-1} .{ }^{1} \mathrm{H}$ NMR (500 MHz: DMSO-d $\left.)_{6}\right): 4.40$ (s, 1H, H4), 5.08 (s, 2H, $\mathrm{CH}_{2}$ ), 5.80 (s, 2H, $\left.\mathrm{CH}_{2}\right), 6.95\left(\mathrm{~d}, J=8.5 \mathrm{~Hz}, 2 \mathrm{H}, \mathrm{H} 3^{\prime}, \mathrm{H} 5^{\prime}\right),\left(\mathrm{d}, J=8.5 \mathrm{~Hz}, 2 \mathrm{H}, \mathrm{H} 2^{\prime}, \mathrm{H} 6\right.$ '), $7.37\left(\mathrm{~s}, 2 \mathrm{H}, \mathrm{NH}_{2}\right)$, 7.45-7.50 (m, 3H, H7, H9, H4"), 7.57 (d, J=8.0 Hz, 2H, H3", H5"), 7.71 (t, J= $7.8 \mathrm{~Hz}, 1 \mathrm{H}$, H8), 7.89 (d, $J=7.8 \mathrm{~Hz}, 1 \mathrm{H}, \mathrm{H} 10), 8.22$ (s, $1 \mathrm{H}$, triazole). ${ }^{13} \mathrm{C}$ NMR (125 MHz: DMSO-d 6 ): 36.2 (C4), 51.2 (benzyl $\left.\mathrm{CH}_{2}\right), 58.1(\mathrm{C} 3), 61.0\left(\mathrm{OCH}_{2}\right), 104.3$ (4H-pyran), $113.0\left(\mathrm{C}^{\prime}\right.$ ' and 5'), 114.7 (coumarin), 116.6 (coumarin), $119.4(\mathrm{CN}), 122.5$ (triazole), 124.7 (Ar), 125.3 (Ar), 128.7 (Ar), 128.8 (Ar), 129.0 (Ar), 130.6 (Ar), 132.9 (Ar), 135.8 (Ar), 135.9 (Ar), 143.0 (triazole), 152.1 (C4'), 153.2 (coumarin, C-O), 157.1 (C2), 158.0 (4H-pyran, C-O), 159.6 $(\mathrm{C}=\mathrm{O}(\mathrm{C} 5))$. Anal. calcd. for $\mathrm{C}_{29} \mathrm{H}_{19} \mathrm{Cl}_{2} \mathrm{~N}_{5} \mathrm{O}_{4}: \mathrm{C}, 60.85 ; \mathrm{H}, 3.35 ; \mathrm{N}, 12.24$. Found: $\mathrm{C}, 60.70$; H, 3.46; N, 12.41 . 
2-Amino-4-(4-((1-(4-bromobenzyl)-1H-1,2,3-triazol-4-yl)methoxy)phenyl)-5-oxo-4H,5Hpyrano[3,2-c]chromene-3-carbonitrile (10g)

Yield: $80 \%$, white crystals, mp 222-224 ${ }^{\circ} \mathrm{C}$. IR (KBr): 3425, 3349, 2197, 1713, 1673, 1605 $\mathrm{cm}^{-1} .{ }^{1} \mathrm{H}$ NMR (500 MHz: DMSO-d $): 4.40(\mathrm{~s}, 1 \mathrm{H}, \mathrm{H} 4), 5.10\left(\mathrm{~s}, 2 \mathrm{H}, \mathrm{CH}_{2}\right), 5.59\left(\mathrm{~s}, 2 \mathrm{H}, \mathrm{CH}_{2}\right)$, $6.96\left(\mathrm{~d}, J=8.4 \mathrm{~Hz}, 2 \mathrm{H}, \mathrm{H} 3^{\prime}, \mathrm{H} 5^{\prime}\right), 7.18$ (d, $\left.J=8.4 \mathrm{~Hz}, 2 \mathrm{H}, \mathrm{H} 2^{\prime}, \mathrm{H} 6^{\prime}\right), 7.27$ (d, $J=8.0 \mathrm{~Hz}, 2 \mathrm{H}$, H2", H6"), 7.39 (s, 2H, NH 2$), 7.45$ (d, J=8.0 Hz, 1H, H7), 7.48 (t, J=8.0 Hz, 1H, H9), 7.56 (d, $J=8.0 \mathrm{~Hz}, 2 \mathrm{H}, \mathrm{H} 3 ", \mathrm{H} 5 "), 7.70$ (t, $J=8.0 \mathrm{~Hz}, 1 \mathrm{H}, \mathrm{H} 8), 7.89$ (t, $J=8.0 \mathrm{~Hz}, 1 \mathrm{H}, \mathrm{H} 10)$, 8.28 (s, $1 \mathrm{H}$, triazole). ${ }^{13} \mathrm{C}$ NMR (125 MHz: DMSO- $\left.d_{6}\right): 36.1$ (C4), 52.1 (benzyl $\mathrm{CH}_{2}$ ), 58.1 (C3), $61.1\left(\mathrm{OCH}_{2}\right), 104.2$ (4H-pyran), 113.0 (C3' and 5'), 114.6 (coumarin), 116.5 (coumarin), $119.3(\mathrm{CN}), 121.4$ (triazole), 122.4 (Ar), 124.7 (Ar), 128.8 (Ar), 129.7 (Ar), 130.2 (Ar), 131.7 (Ar), 132.9 (Ar), 135.4 (Ar), 135.8 (Ar), 143.1 (triazole), 152.1 (C4'), 153.1 (coumarin, C-O), 157.1 (C2), 157.9 (4H-pyran, C-O), 159.5 (C=O (C5)). Anal. calcd. for $\mathrm{C}_{29} \mathrm{H}_{20} \mathrm{BrN}_{5} \mathrm{O}_{4}:$ C, 59.81; H, 3.46; N, 12.02. Found: C, 59.68; H, 3.57; N, 11.87.

\section{2-Amino-4-(4-((1-(4-bromobenzyl)-1 H-1,2,3-triazol-4-yl)methoxy)-3-methoxyphenyl)-5- oxo-4H,5H-pyrano[3,2-c] chromene-3-carbonitrile (10h)}

Yield: $75 \%$, white crystals, mp $235-237^{\circ} \mathrm{C}$. IR (KBr): 3370, 3345, 2935, 2193, 1710, 1670, $1607 \mathrm{~cm}^{-1} .{ }^{1} \mathrm{H}$ NMR (500 MHz: DMSO-d $)$ ) 3.69 (s, 3H, $\left.\mathrm{OCH}_{3}\right), 4.41$ (s, 1H, H4), 5.06 (s, 2H, $\left.\mathrm{CH}_{2}\right), 5.59$ (s, 2H, CH 2$), 6.73\left(\mathrm{~d}, J=8.2 \mathrm{~Hz}, 1 \mathrm{H}, \mathrm{H} 5^{\prime}\right), 6.86(\mathrm{~d}, J=1.0 \mathrm{~Hz}, 1 \mathrm{H}, \mathrm{H} 2$ '), 7.04 (dd, $J=8.2,1.0 \mathrm{~Hz}, 1 \mathrm{H}, \mathrm{H} 6$ ') 7.27 (d, $J=8.0 \mathrm{~Hz}, 2 \mathrm{H}, \mathrm{H} 2 ", \mathrm{H} 6 "), 7.38$ (s, 2H, NH2), 7.457.47 (m, 2H, H7, H9), 7.56 (d, J= 8.0 Hz, 2H, H3", H5"), 7.71 (t, $J=8.0 \mathrm{~Hz}, 1 \mathrm{H}, \mathrm{H} 8), 7.89$

(t, $J=8.0 \mathrm{~Hz}, 1 \mathrm{H}, \mathrm{H} 10), 8.26$ (s, $1 \mathrm{H}$, triazole). ${ }^{13} \mathrm{C}$ NMR (125 MHz: DMSO- $\left.d_{6}\right): 36.5(\mathrm{C} 4)$, 52.1 (benzyl $\left.\mathrm{CH}_{2}\right), 55.5(\mathrm{OMe}), 58.0$ (benzyl $\left.\mathrm{CH}_{2}\right), 61.7\left(\mathrm{OCH}_{2}\right), 104.1$ (4H-pyran), 111.8 (C5'), 113.1 (C2'), 113.6 (coumarin), 116.6 (coumarin), 119.5 (CN), 119.6 (Ar), 121.5 (triazole), 122.5 (Ar), 124.7 (Ar), 124.9 (Ar), 130.3 (Ar), 131.7 (Ar), 132.9 (Ar), 135.4 (Ar), 
136.5 (Ar), 143.1 (triazole), 146.6 (C4'), 148.8 (C3'), 152.1 (coumarin, C-O), 153.3 (C2), 158.0 (4H-pyran, C-O), $159.6(\mathrm{C}=\mathrm{O}(\mathrm{C} 5))$. Anal. calcd. for $\mathrm{C}_{30} \mathrm{H}_{22} \mathrm{BrN}_{5} \mathrm{O}_{5}: \mathrm{C}, 58.84 ; \mathrm{H}$, 3.62; N, 11.44. Found: C, 58.69; H, 3.74; N, 11.29. 


\section{Product 10a}
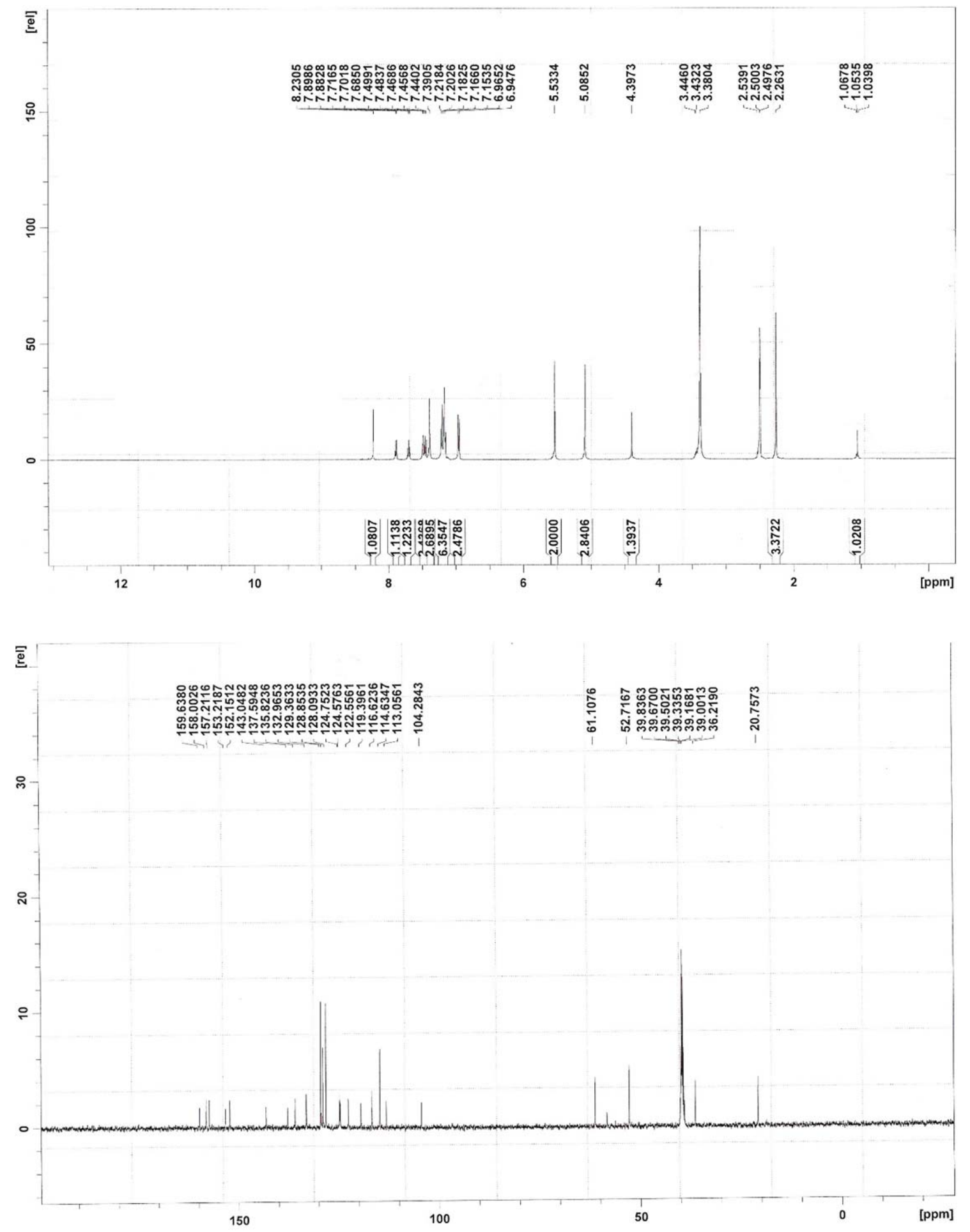


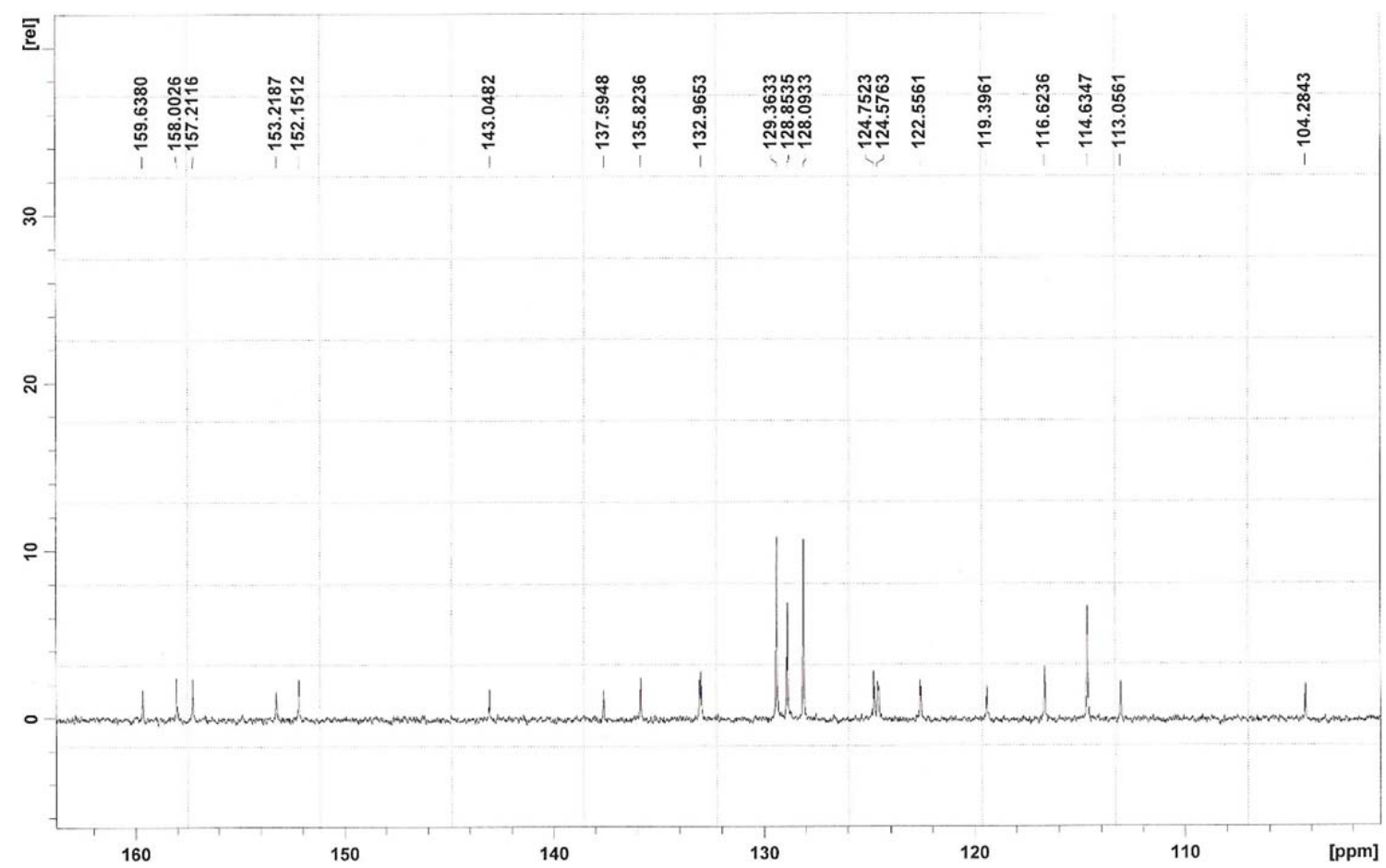

Product 10b

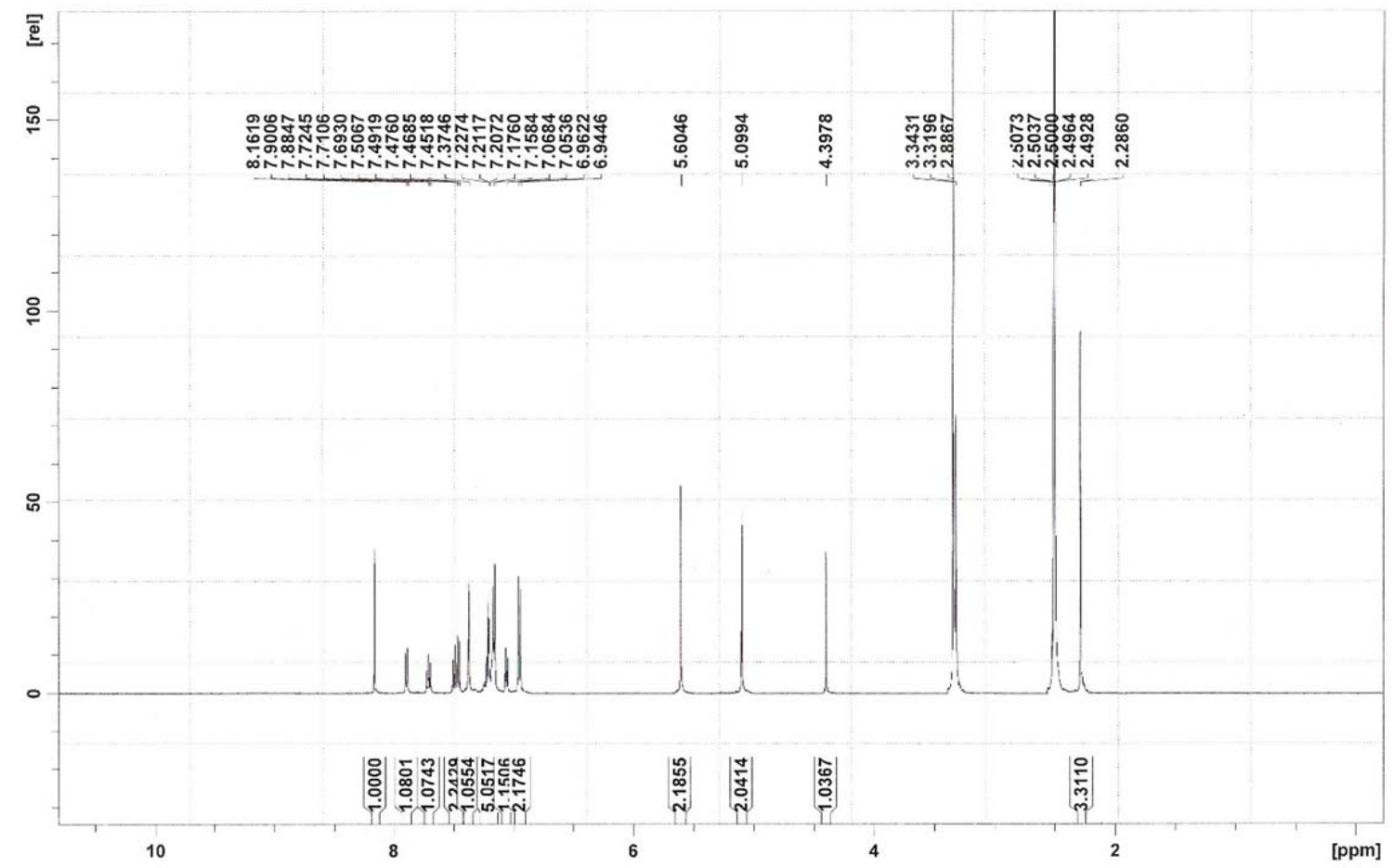




\section{Product 10c}
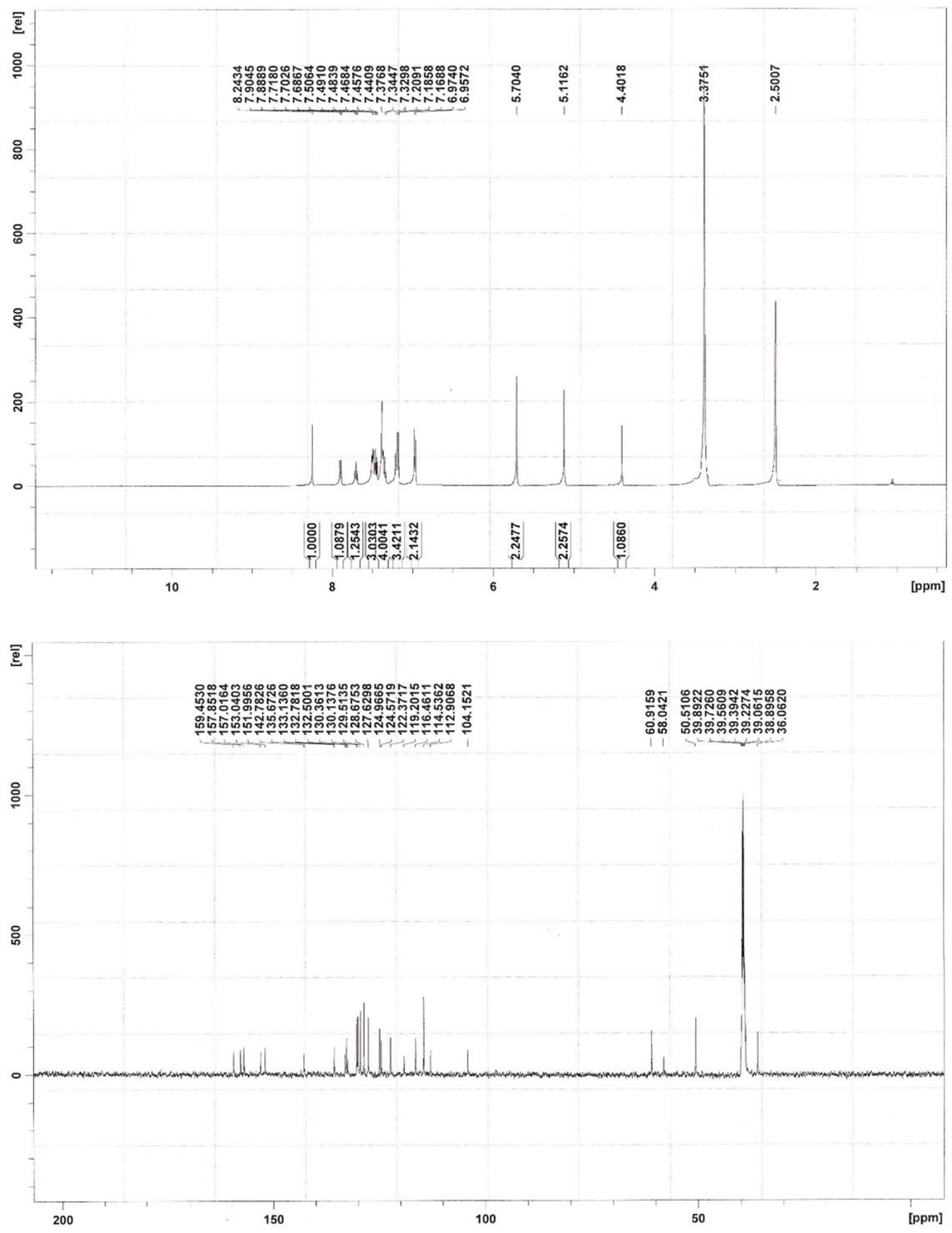


\section{Product 10d}
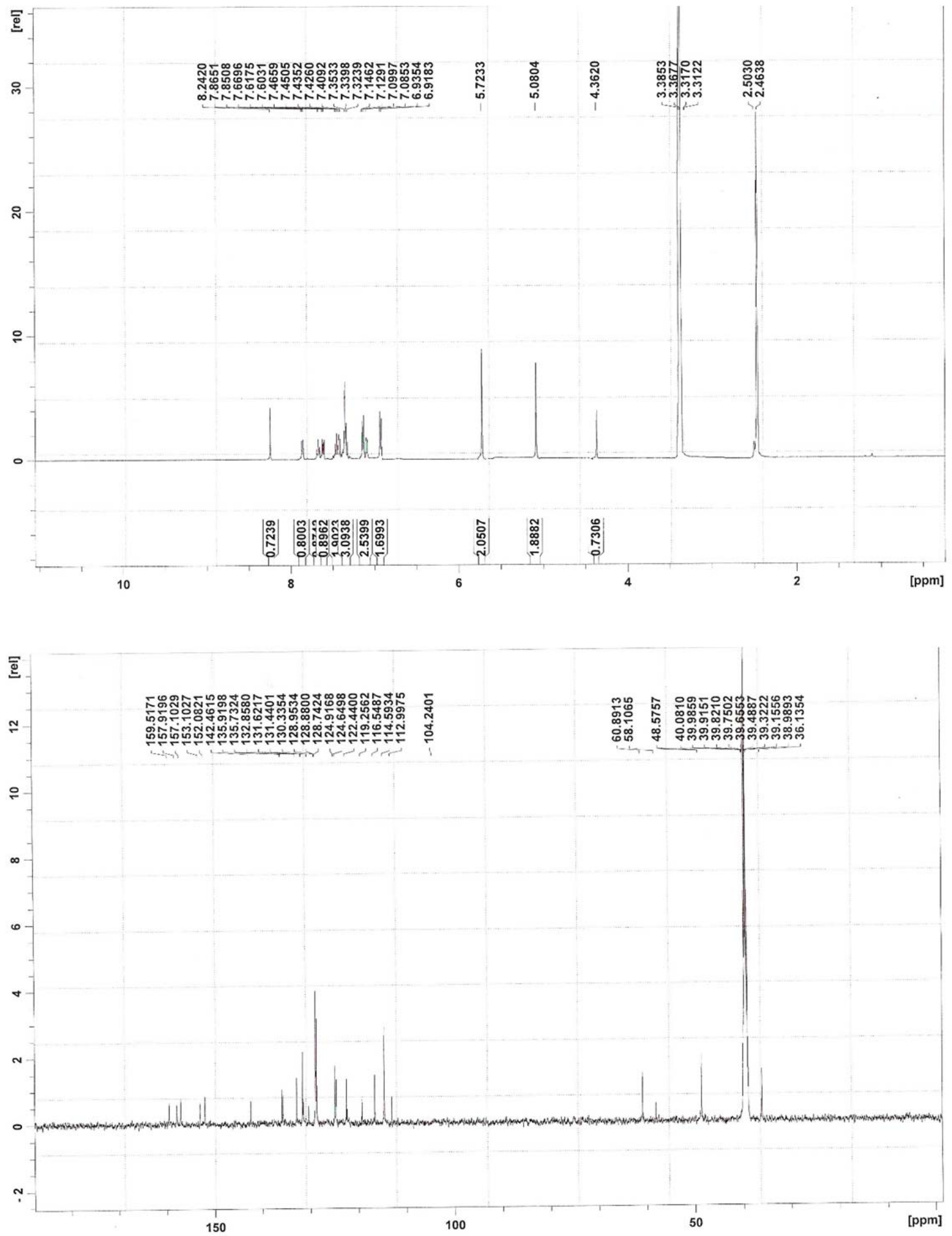


\section{Product 10e}
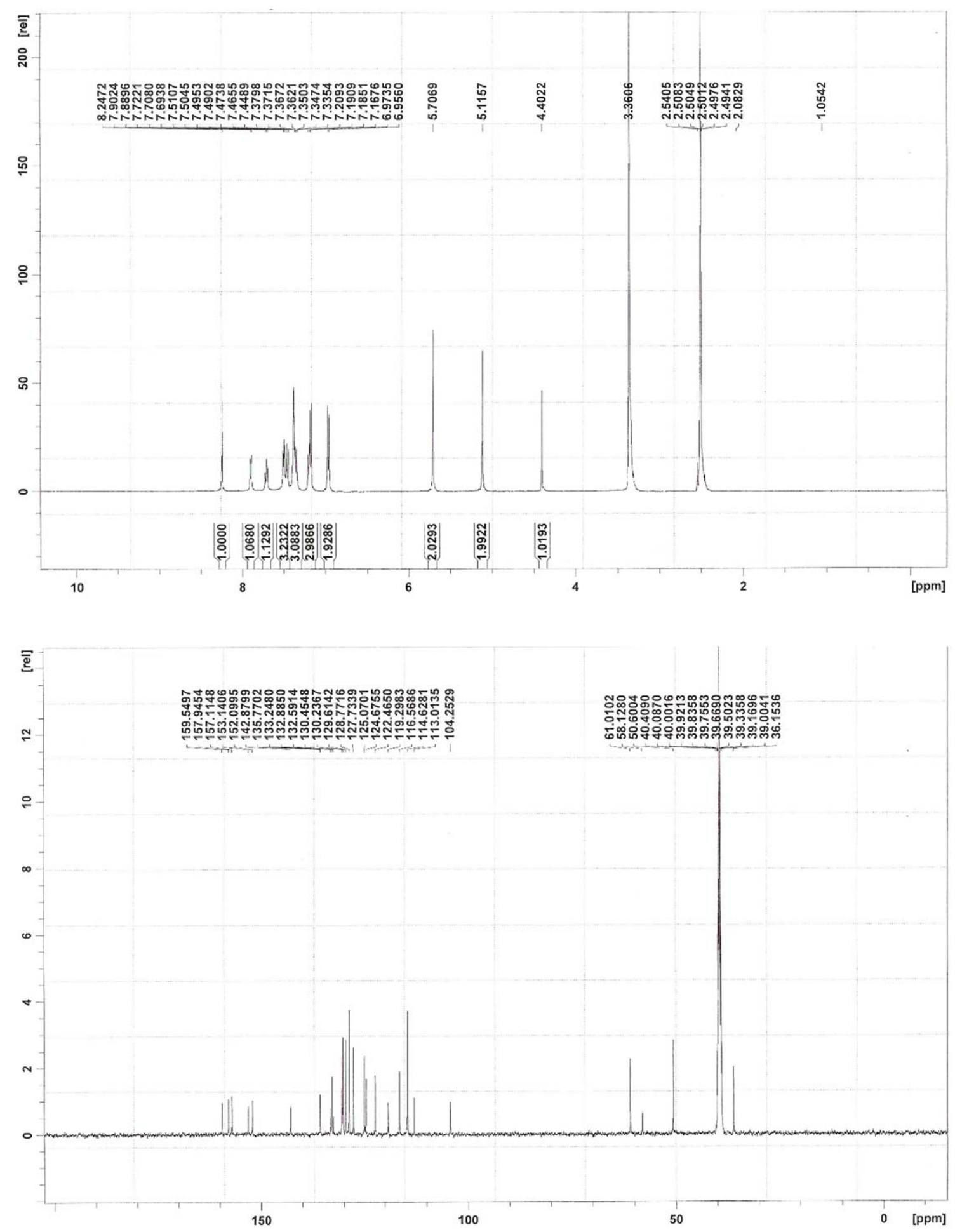


\section{Product 10f}
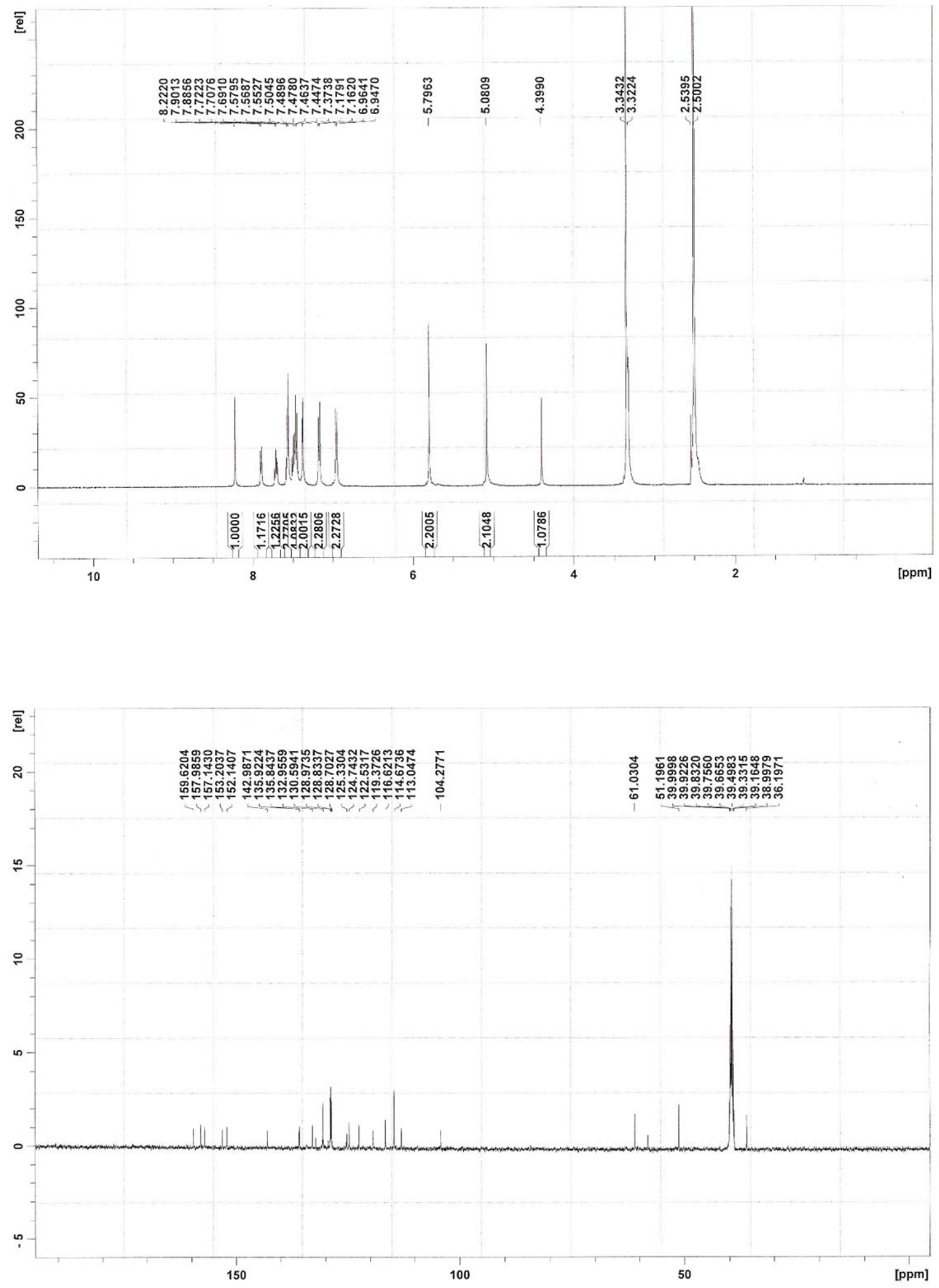


\section{Product 10g}
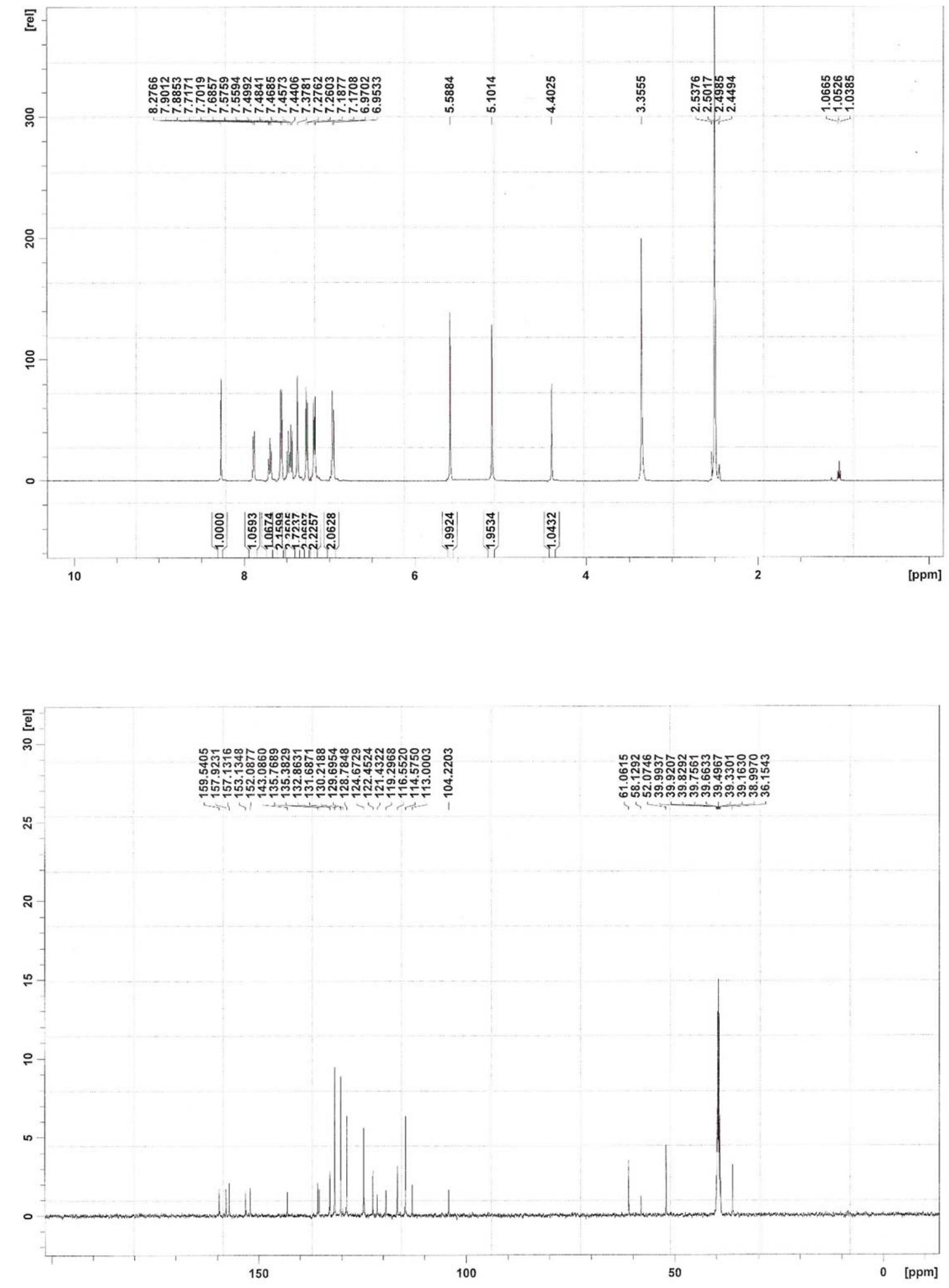


\section{Product 10h}
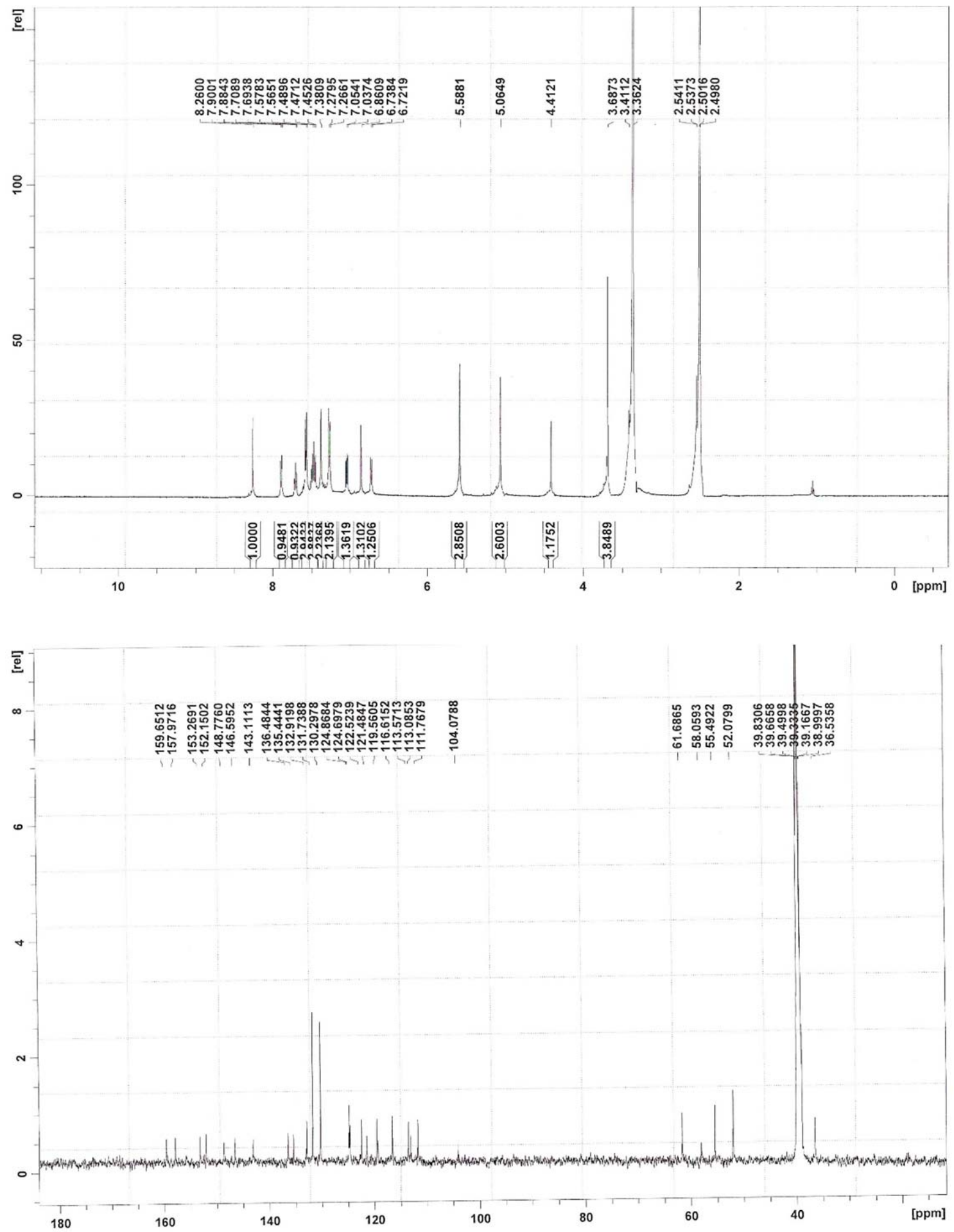


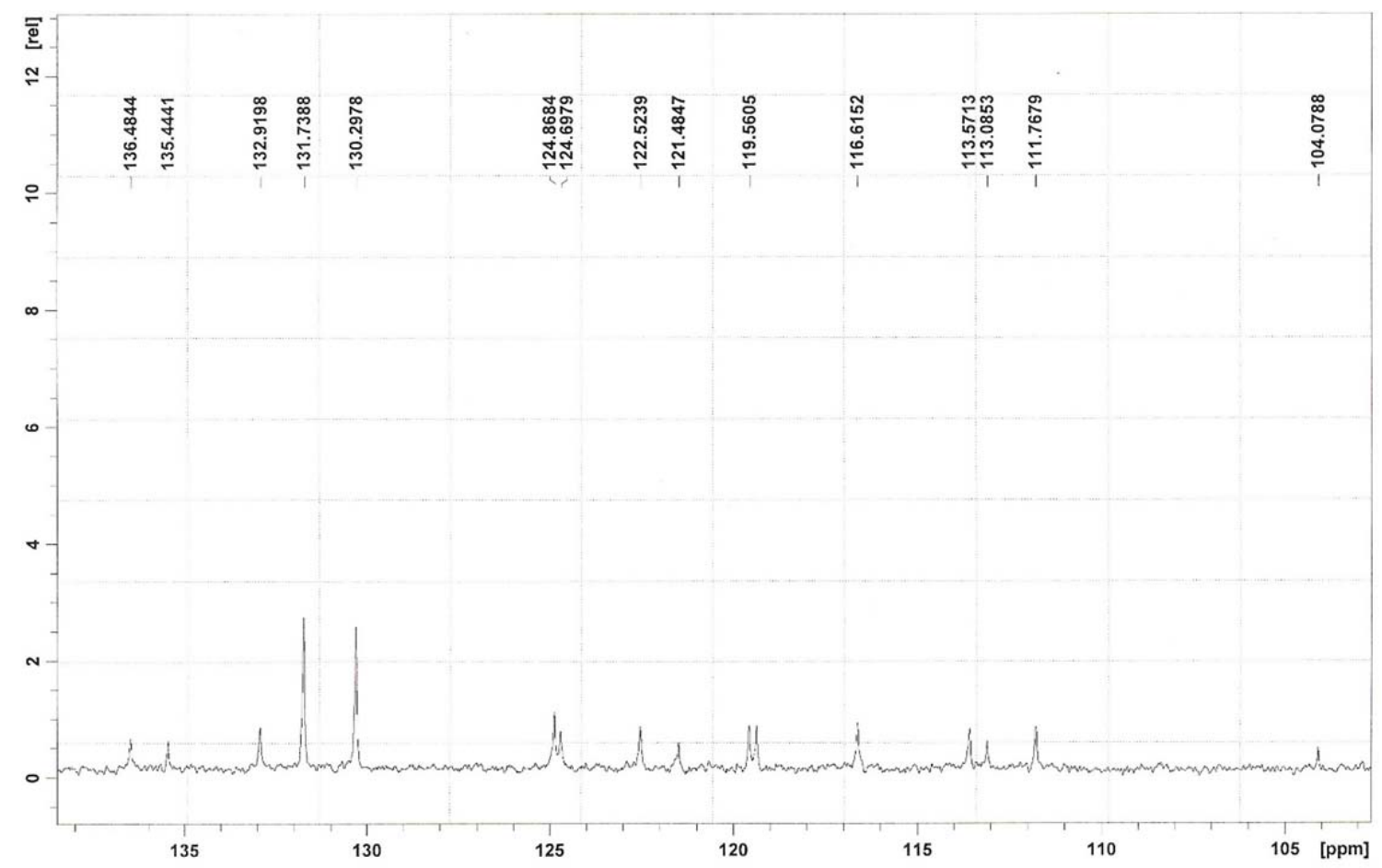




\section{In vitro AChE assay}

Acetylcholinesterase (AChE, E.C. 3.1.1.7, Type V-S, lyophilized powder, from electric eel, 1000 unit), acetylthiocholine iodide (ATCI), and 5,5-dithiobis-(2-nitrobenzoic acid) (DTNB) were obtained from Sigma-Aldrich. Potassium dihydrogen phosphate, dipotassium hydrogen phosphate, potassium hydroxide, and sodium hydrogen carbonate were purchased from Fluka. The solutions of the title compounds were prepared in a mixture of DMSO (5 mL) and methanol (5 mL) and diluted in $0.1 \mathrm{M} \mathrm{KH}_{2} \mathrm{PO}_{4} / \mathrm{K}_{2} \mathrm{HPO}_{4}$ buffer $(\mathrm{pH} 8.0)$ to give the final assay concentrations. All experiments were performed at $25^{\circ} \mathrm{C}$. Six different concentrations were examined for each product in triplicate.

To measure in vitro AChE activity, modified Ellman method was performed ${ }^{[27,33,36]}$ using a 96-well plate reader (BioTek ELx808). Each well contained $50 \mu \mathrm{L}$ potassium phosphate buffer $\left(\mathrm{KH}_{2} \mathrm{PO}_{4} / \mathrm{K}_{2} \mathrm{HPO} 4,0.1 \mathrm{M}, \mathrm{pH} 8\right), 25 \mu \mathrm{L}$ sample dissolved in $50 \%$ methanol and $50 \%$ DMSO and $25 \mu \mathrm{L}$ enzyme (final concentration $0.22 \mathrm{U} / \mathrm{mL}$ in buffer). After preincubation for $15 \mathrm{~min}$ at room temperature, $125 \mu \mathrm{L}$ DTNB (3 $\mathrm{mM}$ in buffer) was added. Characterization of the hydrolysis of ATCI catalyzed by AChE was performed spectrometrically at $405 \mathrm{~nm}$ followed by the addition of substrate (ATCI $3 \mathrm{mM}$ in water). The change in absorbance was measured at $405 \mathrm{~nm}$ after $40 \mathrm{~min}$. A control experiment was conducted under the same conditions without inhibitor and the blank contained buffer, water, DTNB, and substrate. 